\title{
DANKESREDE, ANLÄSSLICH DER ÜBERREICHUNG DES EHRENMITGLIED-DIPLOMS DER LETTISCHEN AKADEMIE DER WISSENSCHAFTEN (FREIBURG A.BR., 28. MAI 1991)
}

Der Lettischen Akademie der Wissenschaften spreche ich meinen herzlichen Dank aus für die große Ehre, die sie mir erwiesen hat mit meiner Wahl zum Ehrenmitglied. Als diese Nachricht kam, hatte ich das Gefühl, daß meine alte Heimat mir beide Hände entgegenstreckt. Das war ein sehr schönes Gefühl. Und nun haben Sie, Herr Professor Stradiň̌s, mir das schöne Dokument überreicht. Ich danke Ihnen herzlich dafür. Ich möchte einiges erwähnen, was uns verbindet.

Durch Heirat 1928 siedelte ich von Lettland nach Deutschland über. Ich bin nun 63 Jahre hier. Durch diesen Umstand habe ich zwei Heimaten gewonnen: das Land meiner Eltern und lettischen Vorfahren, Lettland, und Deutschland, das Land meines Mannes. Zwei Länder anzugehören mit verschiedenen Sprachen und verschiedener Kultur ist eine große innere Bereichung. Beide Länder stehen meinem Herzen nahe.

Jetzt etwas von der Wissenschaft. Die Arbeit meines Mannes hat mich von Anfang an fasziniert. Die Makromoleküle waren eine große Entdeckung. Mein Mann ist 1926 von Zürich nach Freiburg berufen worden und hat sich hier ganz der Ausbreitung der makromolekularen Chemie gewidmet. Ich selber kam von der Biologie, habe in Berlin studiert und bei dem Pflanzenphysiologen Gottlieb Haberlandt promoviert mit einer Arbeit über die Wundheilung an Blättern. Wenn man so eng mit der Zelle und ihren Verwandlungen befaßt ist, so interessiert man sich natürlich auch sehr für ihren Aufbau. Damals, zu Beginn der zwanziger Jahre, konnte man noch nicht bis zu den kleinsten Einheiten vordringen, man hatte sehr geistvolle Hypothesen über den Feinbau der Zelle. Aber als dann die Makromoleküle auf den Plan traten, wurde eine neue Welt erschlossen. Heute ist die makromolekulare Chemie in Gestalt der Molekularbiologie in Biologie und Medizin eingedrungen. Ferner auch in Physik und Mathematik, und sie hat auch der Philosophie Impulse gegeben. Es war aber viel 
Arbeit, die neue Anschauung in der Chemie durchzusetzen. Lange Zeit war die Fachwelt skeptisch. Nach dem Nobelpreis für meinen Mann 1953 gab es dann allgemeine Anerkennung, und die makromolekulare Chemie entwickelte sich zu einem großen neuen Zweig der organischen Chemie.

Dazu möchte ich bemerken, daß beim Aufbau des Fundaments dieser heute so umfangreichen Wissenschaft eine ganze Portion lettischer Kraft, lettischer Interessen und Kräfte verwandt worden ist, die ich ererbt und mitgebracht habe und in die Arbeit meines Mannes in der makromolekularen Wissenschaft verwenden konnte.

In späteren Jahren gab es eine neue Aufgabe: Arbeit für die UNESCO; dazu wird die Lettische Akademie der Wissenschaften etwas beitragen können. Ich wurde am 25. Mai 1961 in die Deutsche UNESCO-Kommission gewählt als Vertreterin des Deutschen Akademikerinnenbundes. In dessen Auftrag hatte ich anfangs mit Ausbildungsfragen für Frauen zu tun und verwandten Themen. Als Naturwissenschaftlerin interessierte mich natürlich auch das naturwissenschaftliche Programm der UNESCO. Darin fanden sich neben einigen kleinen Themen zwei umfassende Weltprogramme: die Hydrologie, also die Erkundung der Wasservorräte der Erde, und die Ozeanographie, die schon in Blüte war, bevor die UNESCO ihre Tätigkeit begonnen hatte, und die nun sehr gefördert werden konnte.

Gegenüber so viel Wasser fehlte die Erde mit ihrem Pflanzenkleid und der Tierwelt. Schon damals hatte der Naturschutz ganz dringende Probleme. Wir begannen unsere Arbeit auf diesem Gebiet mit dem Vorschlag einer umfassenden Resolution, die von der Generalkonferenz der UNESCO 1964 angenommen wurde. Daraf baute sich die weitere Arbeit auf. 1970 nahm die Generalkonferenz der UNESCO ein umfassendes Weltprogramm mit dem Titel "Der Mensch und die Biosphäre" an. Darin wurden als Arbeitsgebiete die Probleme der Nutzung und Erhaltung der Biosphäre festgelegt, und zwar wurden alle Gürtel der Erde von den Tropen bis zur Arktis erfaßt. Eine der Hauptaufgaben dieses Programms ist die Festlegung von Biosphären-Reservaten. Das sind Naturparks und ähnliche Flächen, die die natürliche Vegetation und Tierwelt erhalten, und zu Forschungszwecken und neuen Züchtungen für Ernährungszwecke zur Verfügung stehen, sowie natürlich auch zur Freude der Menschen an den Schönheiten der Natur. Es sind bisher rund um die Erde 300 solche Reservate ausgewiesen. Neun davon befinden sich in Deutschland, wobei die der früheren DDR mitgezählt sind, die recht gut erhalten sind. Im Programm "Der Mensch und die Biosphäre" arbeiten heute hundert Mitgliedstaaten der UNESCO mit. 
Neben diesen praktischen Zielen wurde auch eine Zielsetzung angenommen, die einen besonderen Charakter hat: Ich möchte sie hier im Wortlaut zitieren: "Die Idee einer persönlichen Erfüllung für den Menschen in seiner Partnerschaft mit der Natur soll gefördert werden und seine Verantwortung für dieselbe gestärkt werden." (Zielsetzung 7e des Programms der UNESCO, "Der Mensch und die Biosphäre")

Eine harmonische Partnerschaft des Menschen mit der Natur - wie diese Zielsetzung zitiert wurde vom Generaldirektor der UNESCO, Federico Mayor, gelegentlich seiner Rede zum 40. Geburtstag der Deutschen UNESCO-Kommission vor einem Jahr in der Pauluskirsche in Frankfurt a.M. - diese Partnerschaft bedeutet die persönliche Beziehung des Menschen zur Natur, die Bestätigung seiner Mitgeschöpflichkeit, ein Geben und ein Nehmen mit der Achtung des Partners. Diese Idee hat Wurzeln geschlagen in der Biosphären-Arbeit der UNESCO, und auch diese Arbeit enthält allerlei lettische Kräfte. Ich kann vielleicht nur gerade erwähnen, woher diese Interessen und Kräfte kamen: mein Großvater war Förster in Ropaži, 40 km nördlich von Riga. Ich hoffe, daß weitere Biologen von Lettland hier werden mitmachen können.

Noch einige Worte zur UNESCO: Sie ist bekanntlich die Kulturorganisation der Vereinten Nationen, entstanden auf dem Höhepunkt des Krieges 1942, und zwar in England im Kreise von Julian Huxley, dem berühmten englischen Biologen. Dieser dachte, daß man mit Politik und Wirtschaft allein keine dauernde Ordnung auf der Welt erzielen kann. Er meinte, daß eine Mobilisierung des geistigen Potentials der Menschheit notwendig sei, damit eine dritte Weltmacht, die Weltmacht des Geistes, neben den beiden Weltmächten der Politik und der Wirtschaft entstehen kann. Die UNESCO wurde ein Exponent dieser Idee, in ihr laufen alle Fäden zusammen aus allen Kulturen der Welt. Nach den Erfahrung der letzten Monate ist ihr eine weitere Entwicklung zu wünschen und diese von uns allen zu unterstützen.

Herr Professor Stradinš, nehmen Sie bitte nochmals meinen herzlichen Dank entgegen. Der Lettischen Akademie der Wissenschaften überbringe ich einen Gruß aus der lettischen Hymne:

Dievs, svēti Latviju! 


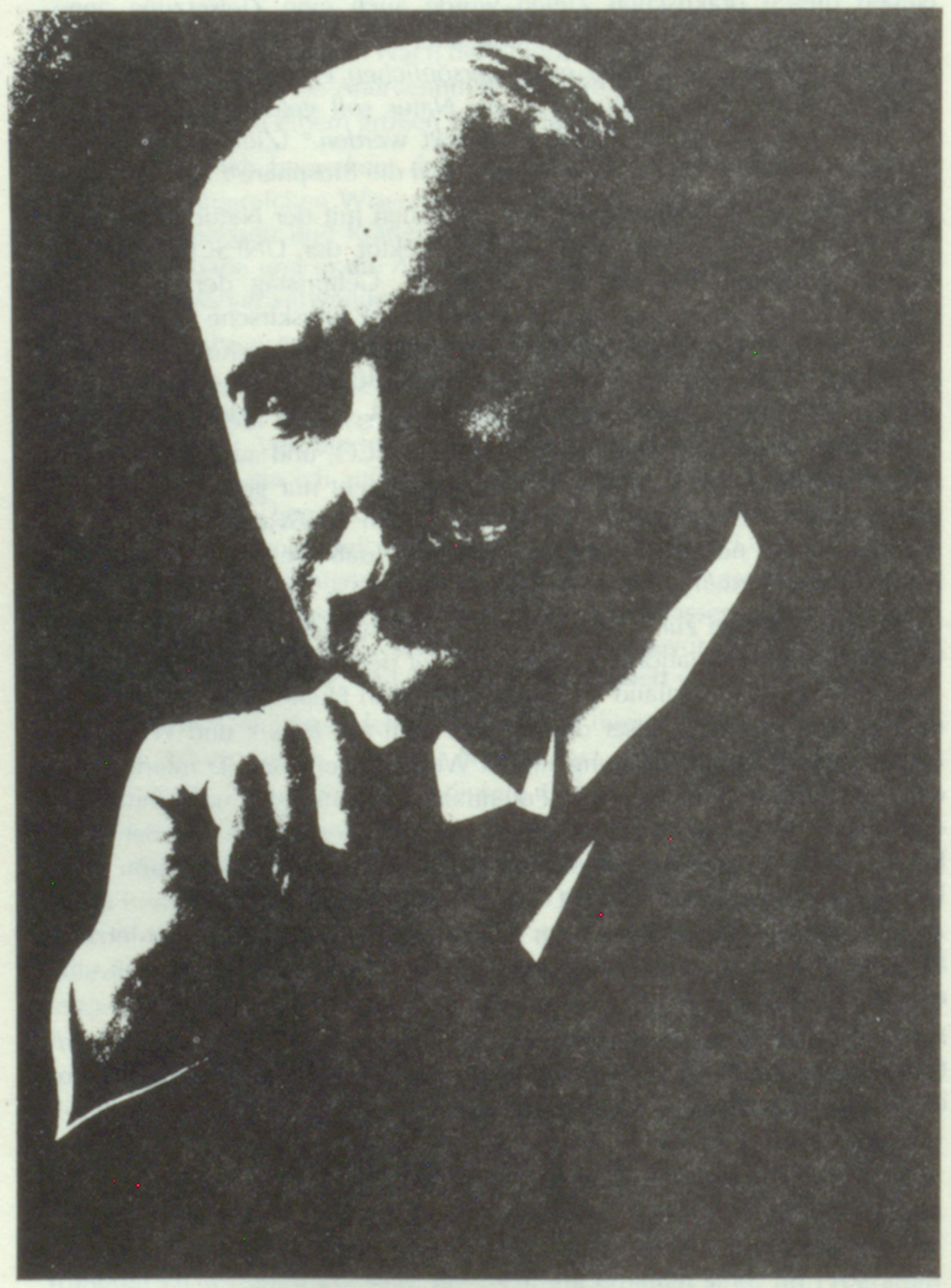

Dr. med. Oskar Woit (1866-1959). 1925 